\title{
Structural Relaxation in $\mathrm{Fe}_{78} \mathrm{Nb}_{2} \mathrm{~B}_{20}$ Amorphous Alloy Studied by Mössbauer Spectroscopy
}

\author{
J. Kansy, A. Hanc, J. Rasek, G. Haneczok, L. Pająk, Z. Stokłosa \\ AND P. KWAPULIŃSKI \\ Institute of Materials Science, University of Silesia, Bankowa 12, 40-007 Katowice, Poland
}

\begin{abstract}
It was shown that soft magnetic properties of $\mathrm{Fe}_{78} \mathrm{Nb}_{2} \mathrm{~B}_{20}$ amorphous alloy can be significantly improved by applying 1-h annealing at temperature $623 \mathrm{~K}$ (permeability increases even about 8 times). The Mössbauer Spectroscopy technique indicated that the optimized microstructure (corresponding to the maximum magnetic permeability) is free of iron nanograins and should be attributed to annealing out of free volume and a reduction of internal stresses i.e. to the relaxed amorphous phase.
\end{abstract}

PACS: $75.75 .+\mathrm{a} ; 76.80 .+\mathrm{y}$

\section{Introduction}

For about 20 years it is known that magnetic properties of amorphous alloys based on iron can be significantly improved by applying a suitable thermal annealing at temperature close to the crystallization temperature. The so-called optimization effect is known in literature for different nanocrystalline alloys i.e. finemet type $\mathrm{Fe}-\mathrm{Cu}-\mathrm{Nb}-$ $\mathrm{Si}-\mathrm{B}$ [1], nanoperm type Fe-Cu-Nb-B [2] or hitperm type Fe-Co-Cu-Nb-B as the optimization effect [3-5]. The observed characteristics are usually explained by formation of a nanocrystalline phase i.e. $\alpha$ Fe nanograins embedded into amorphous matrix. Such a microstructure averages out magnetic anisotropy and gives an enhancement of magnetic permeability. However, as it was shown at first time in [6] in some alloys the optimization effect can be observed without formation of iron nanograins and can be attributed to annealing out of free volume (frozen into material during fabrication) and a reduction of internal stresses [6-12]. This effect is especially interesting because it allows obtaining very good soft magnets free of embrittlement - the main disadvantage — of nanostructured magnets. The aim of the present paper is to study the mechanism of the optimization effect in $\mathrm{Fe}_{78} \mathrm{Nb}_{2} \mathrm{~B}_{20}$ alloy. The examinations of pre-crystallization stages by applying Mössbauer spectroscopy should bring better understanding of the mechanism leading to the enhancement of soft magnetic properties.

\section{Experimental}

The examined alloy i.e. $\mathrm{Fe}_{78} \mathrm{Nb}_{2} \mathrm{~B}_{20}$ was obtained by melt spinning technique in the form of strips with thickness and width of about $25 \mu \mathrm{m}$ and $10 \mathrm{~mm}$, respectively. The as quenched ribbons were annealed in vacuum at temperatures ranging from $300 \mathrm{~K}$ to $850 \mathrm{~K}$ for 1 hour. The Mössbauer spectra of the annealed samples were recorded at room temperature in the standard transmission geometry using a constant acceleration drive. The source was ${ }^{57} \mathrm{Co} / \mathrm{Rh}$ with an activity about $50 \mathrm{mCi}$. For the same samples also at room temperature the following properties were measured: i) relative magnetic permeability $\mu_{r}$ at magnetic fields $0.5 \mathrm{~A} / \mathrm{m}$ (HP $4284 \mathrm{~A}$ meter working at frequency of about $1 \mathrm{kHz}$ ), ii) electrical resistivity $\rho$ (four point probe) and iii) X-ray diffraction patterns (Philips Diffractometer X'Pert PW 3040/60).

\section{Mössbauer data analysis}

The Mössbauer spectra were deconvoluted into a set (about 16) of elementary Zeeman sextets by applying a least-square fitting procedure. Positions of the spectrum lines in each sextet were expressed by the hyperfine structure parameters, i.e. the hyperfine magnetic field $B$, isomer shift of the sextet center $S$ and quadrupole shift (first and sixth lines towards the second to fifth lines) $Q$. This parameter was taken into account because of possible non cubic symmetry of atomic configurations around ${ }^{57} \mathrm{Fe}$. In order to reduce the number of free parameters used in the fitting, a linear correlation between $B$ and $S$ was assumed i.e. $S=S_{0}+\beta B$ (where $S_{0}$ and $\beta$ are constant). Such a correlation was observed in literature for mean values of $B$ and $S[13,14]$ as well as for respective local value of $B$ and $S[14,15]$. The absorption line intensities of each sextet satisfied the condition:

$$
I_{1,6}: I_{2,5}: I_{3,4}=3: R: 1,
$$

where $R$ and the total intensity of each sextets were a free fitting parameters. In amorphous structure of investigated samples one can expect many atomic configurations inducing similar hyperfine fields on ${ }^{57} \mathrm{Fe}$, therefore each value of $B$ related to a sextet was assumed to be likely dispersed. This factor was considered as a broadening of the spectrum lines. So, it was assumed that the width of a line is proportional to the distance of the line from the spectrum center

$$
\Gamma_{\text {broad }}=\left(1+\varepsilon\left|v_{i}\right|\right) \Gamma_{0},
$$

where $v_{i}$ is the position of $i$-th line of sextet, $\Gamma_{0}$ is the nat- 
ural line width and $\varepsilon$ is a constant. The fitted theoretical function took into consideration finite effective thickness of sample $\left(t_{a}\right)$ and was determined with the transmission integral.

Because of a large amount of free fitting parameters, a single sextet parameters have no well defined physical meaning, so in our interpretation we took into account quantities of global character only, such as the mean hyperfine field $B_{m}$, its dispersion $\sigma_{B}, R$ parameter and $\beta$.

\section{Results and discussion}

Figure 1 shows the selected Mössbauer spectra obtained for annealed sample of the $\mathrm{Fe}_{78} \mathrm{Nb}_{2} \mathrm{~B}_{20}$ alloy together with the fits of the data. Beside are inserted respective hyperfine field distributions $P(B)$, where $P$ is the total intensity of a sextet relating to a given value of $B$.

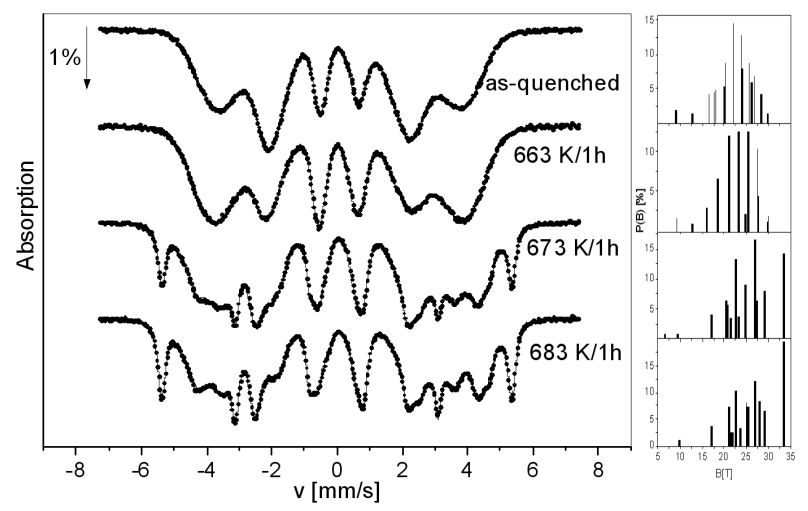

Fig. 1. The Mössbauer spectra of selected $\mathrm{Fe}_{78} \mathrm{Nb}_{2} \mathrm{~B}_{20}$ samples after annealing at different temperatures (specified in figures) for 1 hour (the solid lines represent fits).

Right - the respective hyperfine field distributions.

On the basis of the obtained distributions $P(B)$ the mean value of hyperfine field $B_{m}$ and the dispersion $\sigma_{B}$ were determined. The main result of the paper is shown in Fig. 2 where we plot relative magnetic permeability $\mu_{r}$, resistivity $\rho$ and Mössbauer parameters $\left(B_{m}, \sigma_{B}\right.$ and $\left.R\right)$ vs. annealing temperature $T_{a}$ for the $\mathrm{Fe}_{78} \mathrm{Nb}_{2} \mathrm{~B}_{20}$ alloy. One can see that the optimization annealing i.e. annealing at the temperature $T_{o p}=623 \mathrm{~K}$ for $1 \mathrm{~h}$ causes an increase of magnetic permeability about 8 times in relation to the as quenched state. Note that resistivity $\rho$ decreases at $T_{a}$ slightly higher than $T_{o p}$. That indicating that crystallization (nanocrystallization) occurs at $T_{a}>T_{o p}$. Indeed, such conclusion was confirmed by XRD patterns (not shown). The first narrow X-ray reflexes on a background of a broad maximum were observed for sample annealed at $673 \mathrm{~K} / 1 \mathrm{~h}$, i.e. about $50 \mathrm{~K}$ above $T_{o p}$.

From Fig. 2 one can see that for $T_{a}<670 \mathrm{~K}, \sigma_{B}$ is almost constant and $B_{m}$ faintly increases. The weak increase of $B_{m}$ is connected with the relaxation of amorphous phase, i.e. with annealing out of free volumes causing a reduction of a mean distance between iron atoms.

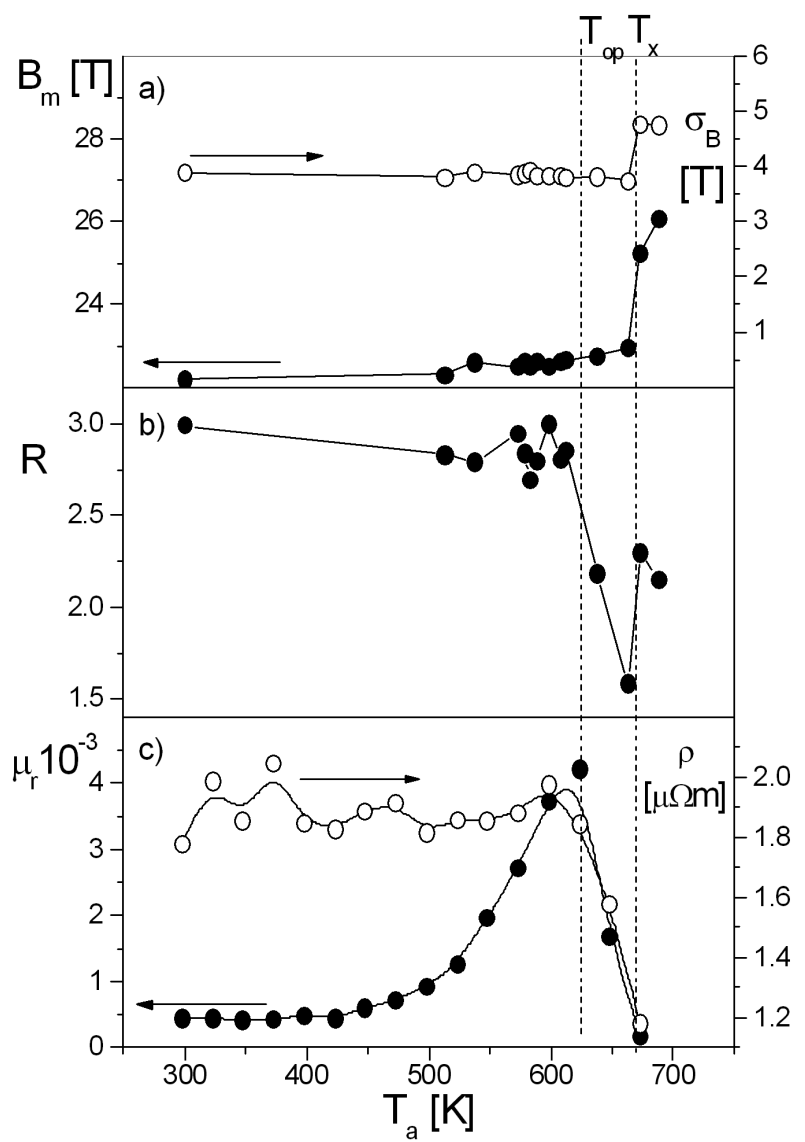

Fig. 2. Different physical quantities determined for the $\mathrm{Fe}_{78} \mathrm{Nb}_{2} \mathrm{~B}_{20}$ amorphous alloys plotted vs. 1-h annealing temperature $T_{a}$. a) The average hyperfine field $B_{m}$ and dispersion $\sigma_{B}$ of the field distribution. b) The ratio $R$ of the intensity of the second (fifth) line to the intensity of the third (fourth) line in each sextet. c) Relative magnetic permeability $\mu_{r}$ and resistivity $\rho ; T_{o p}$ - the 1-h optimization temperature annealing, $T_{x}$ — the crystallization temperature.

The amorphous structure of the material, for $T_{a}<670 \mathrm{~K}$ is confirmed by Mössbauer spectra shown in Fig. 1. The Mössbauer spectrum lines of positions characteristic for $\alpha \mathrm{Fe}$ are observed for the sample annealed at $T_{a}=673 \mathrm{~K}$. This is reflected by steep increase of $B_{m}$ and $\sigma_{B}$ (see Fig. 2a). For the sample annealed at $T_{a}=688 \mathrm{~K}$ the Mössbauer spectrum lines related to $\alpha \mathrm{Fe}$ become more distinct. The XRD spectra obtained for the same sample show that the broad maximum practically disappears. Moreover, the samples in the as quenched state and annealed at $T_{a}<623 \mathrm{~K}$ reveal relatively high magnetic texture. The value of $R$ about 3 (see Fig. $2 \mathrm{~b}$ ) indicates that magnetic field vectors lie mainly in the plane parallel to the surface of sample. Such a texture is usually a characteristic feature of the melt spun ribbons. Formation of the earliest crystallization nucleus causes a drastic reduction of $R$ even below 2. This value suggests that some magnetic moments have changed the orienta- 
tion from parallel to perpendicular to the sample surface. It seems to be a fluctuation due to pre-crystallization stages. For $T_{a}>670 \mathrm{~K}$ (nanocrystallization) the $R$ parameter reaches the value about 2 indicating a disappearance of the texture.

The results presented in Fig. 2 allow concluding that the observed optimization effect cannot be attributed to iron nanostructure. The significant increase of permeability is due to annealing out of free volume and a reduction of internal stresses, as it was discussed in [6-12].

The parameters used in the fits $S_{0}$ and $\beta$ are gathered in Table. Before crystallization $\left(T_{a}<670 \mathrm{~K}\right)$ both parameters $S_{0}$ and $\beta$ fluctuate around their mean values which are specified in second column of Table. However, just after crystallization these parameters change strongly into values of opposite signs. Similar effect was observed also for amorphous alloys based on iron and cobalt in paper [15]. The relative line broadening $\varepsilon$ (see Eq. (2)) was assumed to be the same for all spectra (except $\alpha$ Fe sextet for which $\varepsilon=0$ ) and using the fit procedure we have got $\varepsilon=0.539$ as a mean value.

TABLE

The Isomer Shift parameters $S_{0}$ and $\beta$ (see the text) determined for the $\mathrm{Fe}_{78} \mathrm{Nb}_{2} \mathrm{~B}_{20}$ amorphous alloys.

\begin{tabular}{l|l|l|l}
\hline \hline & Before crystallization & \multicolumn{2}{|c}{ After crystallization } \\
\hline$T_{a}[\mathrm{~K}]$ & $300-663$ & 673 & 688 \\
\hline$S_{0}[\mathrm{~mm} / \mathrm{s}]$ & $-0.126 \pm 0.009^{*}$ & $0.209^{*}$ & $0.247^{*}$ \\
\hline$\beta[\mathrm{mm} / \mathrm{sT}]$ & $(6.8 \pm 0.28) \times 10^{-3}$ & $6.6 \times 10^{-3}$ & $-7.9 \times 10^{-3}$ \\
\hline${ }^{*}$ in relative to $\alpha$-Fe standard. &
\end{tabular}

The main conclusions of the present paper can be summarized as follows: i) magnetic permeability of the $\mathrm{Fe}_{78} \mathrm{Nb}_{2} \mathrm{~B}_{20}$ alloy can be enhanced even 8 times in relation to the as quenched state by applying 1-h annealing at temperature $623 \mathrm{~K}$, ii) the optimized microstructure (corresponding to the optimal soft magnetic properties) is free of iron nanograins and can be attributed to the relaxed amorphous phase.

\section{Acknowledgements}

This work was partially supported by the Polish Ministry of Science and Education under grant No. NN 507460633 .

\section{References}

[1] Y. Yoshizawa, S. Oguma, K. Yamauchi, J. Appl. Phys. 64, 6044 (1988).

[2] A. Makino, T. Hatani, Y. Naitoh, T. Bitoh, A. Inoue, IEEE Trans. Magn. 33, 3793 (1977).

[3] X. Liang, T. Kulik, J. Ferenc, B. Xu, J. Magn. Magn. Mater. 308, 227 (2007).

[4] J. Zbroszczyk, A. Młyńczyk, J. Olszewski, W. Ciurzyńska, M. Hasiak, R. Kolano, J. Lelątko, J. Magn. Magn. Mater. 304, e727 (2006).

[5] R. Hasegawa, J. Magn. Magn. Mater. 304, 187 (2006).

[6] T. Naohara, Philos. Mag. Lett. 78, 229 (1998).

[7] G. Haneczok, J. Rasek, Defects Diffus. Forum 224225, 13 (2004).

[8] G. Haneczok, J.E. Fracckowiak, A. Chrobak, P. Kwapuliński, J. Rasek, Phys. Status Solidi A 202, 2574 (2005).

[9] A. Chrobak, G. Haneczok, Z. Stokłosa, P. Kwapuliński, J. Rasek, G. Chełkowska, Phys. Status Solidi A 196, 248 (2003).

[10] A. Chrobak, G. Haneczok, P. Kwapuliński, D. Chrobak, Z. Stokłosa, J. Rasek, J. Alloys Compd. 423, 77 (2006).

[11] G. Badura, J. Rasek, Z. Stokłosa, P. Kwapuliński, G. Haneczok, J. Lelątko, L. Pająk, J. Alloys Compd. 436, 43 (2007).

[12] Z. Stokłosa, J. Rasek, P. Kwapulińnski, G. Badura, G. Haneczok, J. Magn. Magn. Mater. 304, 700 (2006).

[13] S.M. Dubiel, W. Zinn, Phys. Rev. B 26, 1574 (1982).

[14] A. Błachowski, K. Ruebenbauer, J. Żukrowski, Nukleonika 49, 67 (2004).

[15] J. Olszewski, J. Zbroszczyk, K. Sobczyk, W. Ciurzyńska, P. Brągiel, M. Nabiałek, J. Świerczek, M. Hasiak, A. łukiewska, Acta Phys. Pol. A 114, 1659 (2008). 